\title{
Contextual information in (non) native speech perception in noise
}

\author{
Bin $\mathrm{Li}^{1}$, Ratree Wayland ${ }^{2}$, Caicai Zhang ${ }^{1}$ \\ ${ }^{1}$ Department of Chinese, Translation \& Linguistics, City University of Hong Kong, \\ Hong Kong S.A.R. \\ ${ }^{2}$ Linguistics Program, University of Florida, Gainesville, Florida, U.S.A. \\ https://doi.org/10.36505/ExLing-2010/03/0026/000146
}

\begin{abstract}
Non-native listeners' perception of speech has been shown to be more adversely affected by interference from background noise than native speakers. This study focused on effects of different levels of linguistic information on speech perception in noise. We examined roles of phonetic and contextual cues in sentence perception in babble noise by Chinese learners and native speakers of English. Our sentences varied in degrees of syntactic and semantic acceptability. Results revealed similar patterns of perception in the performance of listener groups. Secondly, listeners benefited more from certain sentence types under different listening conditions.
\end{abstract}

Key words: speech perception in noise, ESL/EFL learners, sentence perception

\section{Introduction}

Everyday speech communication is often characterized by noise, which adversely affects the understanding of speech. Studies showed that non-native listeners are less tolerant with the degradation of speech than native listeners (Roussohatzaki, Florentine 1990). To alleviate the communication burden on the listener, researchers suggested that the talker use a clear speaking style (Picheny et. al. 1985, Hazan, Simpson 1998, Bradlow, Bent 2002, Wayland et. al. 2006). Few previous studies, however, explored roles of higher-level linguistic information in perceiving speech in noise.

\section{The current study}

We aimed at examining effects of linguistic information at various levels in perceiving sentences with varying degrees of acceptability under different listening conditions.

\section{Stimuli}

74 English sentences with varying syntactic and semantic properties were created. All were evaluated by 6 adult native speakers of English (NE) for their acceptability on a scale of 5 . Sentences were then grouped in to 5 types: context dependent (CD)sentences with semantically related and predictable keywords, e.g. The boat sank in the storm; sentences containing context neutral (CN) keywords, e.g. A friend came for lunch; sentences with syntactically marked (SY) structures, e.g. Up the

ExLing 2010: Proceedings of 3rd Tutorial and Research Workshop on Experimental Linguistics, 25-27 August, Athens, Greece 
hill, Mary ran; and semantically marked (SE) sentences with unpredictable collocation, e.g. They are drinking oil; and lastly, syntactically or semantically unacceptable (U) sentences, e.g. They liked in cold water. Production of all sentences was recorded from 2 adult NE speakers, which was digitized and normalized for intensity and then mixed with babble noise at two Signal-to-Noise (SNR) ratios: $-4 \mathrm{~dB}$ and $-8 \mathrm{~dB}$. Altogether $148(74 \times 2)$ test sentences were generated.

\section{Listeners}

70 adult Chinese EFL/ESL learners were recruited with normal hearing ability from China's mainland (MN) and Hong Kong (HK) participated. All were full-time university students, and had taken TOEFL or IELTS. 10 adult NE speakers, none of whom participated in sentence preparation, were recruited as our control group. Listeners were aged between 18-35 years.

\section{Procedure}

Listeners were instructed to listen to each sentence as many times as they wished before rewriting. They also completed acceptability evaluation on all sentences.

\section{Results and Discussion}

Mean percentages correct for keywords rewriting were calculated and compared across listener groups, sentence types and listening conditions.

\section{English proficiency}

Self-reported scores of IELTS and TOEFL (TOEFL scores were converted to IELTS bands for comparison) helped group Chinese listeners into 4 groups: Band 5, Band 6, Band 7, Band 8. Mainland listeners had an average score of 7.13 whereas Hong Kong listeners' average score was significantly lower at $6.48(\mathrm{p}<0.001)$. Listeners' performance was generally positively correlated with their English proficiency (Table 1). A closer look into each SNR revealed that the correlation was stronger under the $-4 \mathrm{~dB}$ SNR condition than under the $-8 \mathrm{~dB}$ one, suggesting a less robust advantage of language proficiency in more adverse listening conditions for non-native listeners.

\section{Listening conditions}

All listeners did significantly better under $-4 \mathrm{~dB}$ SNR condition than under the $-8 \mathrm{~dB}$ one $(\mathrm{p}<0.01$ for all) (Table 1, Fig. 1). NE speakers scored highest in both conditions and MN listeners outperformed HK listeners ( $\mathrm{p}<.05$ for $-8 \mathrm{~dB}$ SNR and $\mathrm{p}<.01$ for all other conditions). As reported earlier, MN listeners were more proficiency in English than HK listeners. This suggested again that the adverse effects of noise on speech perception are more significant 
Contextual information in (non) native speech perception in noise 103

for less proficient learners. When noise reached a level where even NE speakers found perception difficult (41.05\% correct rewriting), the difference between learners at various proficiency levels started to diminish.

\section{Sentence types}

Our acceptable sentences differed in keywords predictabilities and thus were divided into CD (context dependent), CN (context neutral), SY (marked syntactic structures), and SE (marked semantic information). Both Chinese and NE listeners evaluated all sentences on their acceptability, results of which were highly correlated (Table 2). Rewriting results showed that SE, $\mathrm{CD}, \mathrm{CN}$ types as a group were significantly better than SY and $\mathrm{U}$ for all listeners (all $\mathrm{p}<0.02$ ) under $-4 \mathrm{~dB}$ SNR condition (Fig. 1). Under $-8 \mathrm{~dB}, \mathrm{NE}$ listeners did numerically better with the $\mathrm{CD}$ and $\mathrm{CN}$ types, but poorer with $\mathrm{SE}$. On the other hand, for Chinese listeners, $\mathrm{CN}$ was better than the group of SE, SY and U, which was better than CN (all p<0.04). So when noise level was relatively low, e.g. $-4 \mathrm{~dB}$ SNR, all listeners found SE sentences easier despite its lower degree of acceptability. However, under -8dB SNR condition, such facilitative effect diminished for even NE speakers (though not significantly worse than others, $p>0.05$ ). Another interesting finding was the greater deteriorating performance of Chinese listeners with the CD type than SE under -8dB SNR condition. This may be due to their greater dependence on semantic information than on syntactic structures under $-4 \mathrm{~dB}$ SNR condition. Under -8dB SNR condition, however, Chinese listeners may rely more on phonetic cues. The effects of predictable keywords in CD sentences were only consistently beneficial to NE listeners.

Table 1. Mean percent correct (Std. error of mean) of keywords rewriting.

\begin{tabular}{lllll}
\hline \hline Chinese listeners & SNR -4dB & \multicolumn{3}{l}{ SNR -8dB } \\
\hline Band 8 & 49.95 & $(1.72)$ & 23.34 & $(1.50)$ \\
Band 7 & 48.19 & $(1.82)$ & 24.38 & $(1.42)$ \\
Band 6 & 45.28 & $(1.75)$ & 19.58 & $(1.28)$ \\
Band 5 & 41.77 & $(1.48)$ & 18.40 & $(1.12)$ \\
\hline NE speakers & 75.65 & $(1.59)$ & 41.05 & $(1.96)$ \\
\hline \hline
\end{tabular}

Table 2. Mean (Std. error of mean) sentence acceptability, 1meaning totally acceptable.

\begin{tabular}{|c|c|c|c|}
\hline & Chinese listeners & NE listeners & Correlation \\
\hline $\mathrm{CN}$ & $2.054(.010)$ & $1.173 \quad(.014)$ & $\mathrm{r}=0.85, \mathrm{p}<0.001$ \\
\hline $\mathrm{CD}$ & $2.216 \quad(.012)$ & $1.334 \quad(.027)$ & \\
\hline SY & $2.498 \quad(.011)$ & $2.176(.023)$ & \\
\hline SE & $2.614 \quad(.014)$ & $2.835 \quad(.026)$ & \\
\hline $\mathrm{U}$ & $3.093 \quad(.009)$ & $3.880 \quad(.012)$ & \\
\hline
\end{tabular}




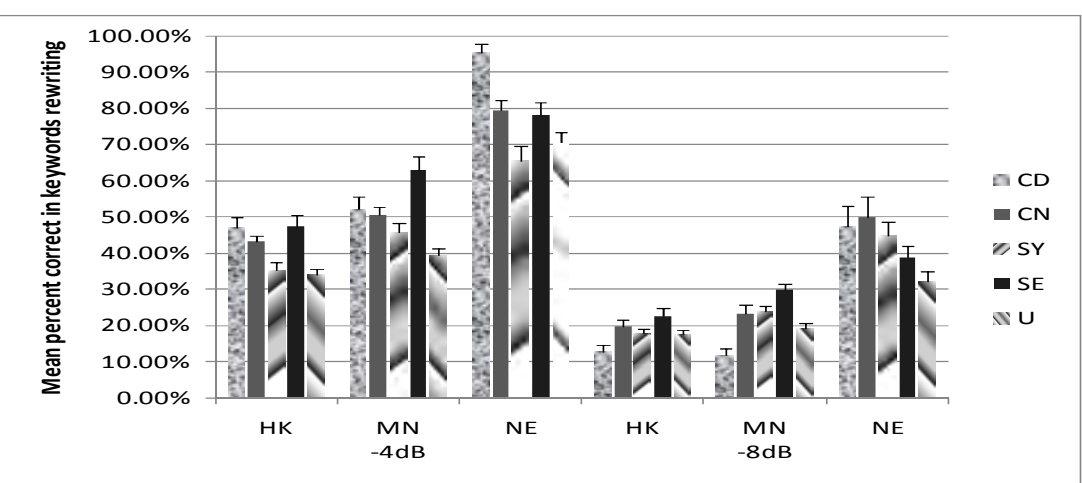

Figure 1. Mean percent correct in keywords rewriting by listener groups and sentence types.

\section{Conclusion}

Our study aimed to investigate whether perception of impaired speech by listeners is influenced by their limited usage of the contextual cues or their inability to process speech acoustic signals. We found that listeners' abilities in using semantic or syntactic information in speech perception were commensurate with their proficiency in the target language. Second, semantic information had a stronger facilitating effect on speech perception in noise than syntactic information for both native and non-native listeners. Thirdly, the facilitating effects may be reduced by noise at higher SNRs, whereas acoustic-phonetic information may become more useful in such situations.

\section{Acknowledgements}

This study was supported by SRG Grant \#7002191 of City University of Hong Kong. We thank Mr. Peng Yukang for his help in data collection.

\section{References}

Bradlow, A.R., Bent, T. 2002. The clear speech effect for non-native listeners. J. Acoust. Soc. Am. 112 (1), 272-284.

Hazan, V., Simpson, A. 1998. The effect of cue enhancement on the intelligibility of nonsense word and sentence materials presented in noise. Sp. Comm. 24, 211-226.

Picheny, M.A., Durlach, N.I., Braida L.D. 1985. Speaking clearly for the hard of hearing I. J Speech Hear Res. 28 (1), 96-103.

Roussohatzaki, M., Florentine, M. 1990. Perception of American-English in noise by Greek listeners, J. Acoust. Soc. Am. 87, S72.

Wayland, R, Li, B., Csonka, L., Harnsberger, J. 2006. Effects of experience on speech perception in noise. J. Acoust. Soc. Am. 119, 3422. 